\title{
Surface-Enhanced Raman Scattering Study of Silver Nanoparticles Prepared by Using MC as a Template
}

\author{
Yudong Lu, ${ }^{1,2}$ Shanyuan Feng, ${ }^{2}$ Xueyun Liu, ${ }^{2}$ and Lihui Chen ${ }^{1}$ \\ ${ }^{1}$ College of Material Engineering, Fujian Agriculture and Forestry University, Fuzhou, Fujian 350002, China \\ ${ }^{2}$ College of Chemistry and Chemical Engineering, Fujian Normal University, Fuzhou, Fujian 350007, China \\ Correspondence should be addressed to Lihui Chen; lihuichen66@126.com
}

Received 26 September 2013; Revised 3 December 2013; Accepted 4 December 2013

Academic Editor: Miguel A. Correa-Duarte

Copyright (c) 2013 Yudong Lu et al. This is an open access article distributed under the Creative Commons Attribution License, which permits unrestricted use, distribution, and reproduction in any medium, provided the original work is properly cited.

\begin{abstract}
A good Ag-based SERS substrate has been prepared by one-pot reaction using methyl cellulose as a template. Effects of methyl cellulose concentration, silver ammonia chloride solution concentration, reaction duration, and reducing agent on silver nanoparticles were discussed in this paper. The performance of the obtained Ag nanoparticles was characterized by UV-visible spectroscopy, transmission electron microscopy, and surface-enhanced Raman spectroscopy. Results show that the reducing agent plays a crucial role in the performance of silver nanoparticles. Optimum preparation conditions of synthesis of SERS substrates were as follows: $10 \mathrm{mM}$ silver ammonia chloride and $0.2 \% \mathrm{MC}$ at $75^{\circ} \mathrm{C}$, reducing in $0.2 \%$ reducing agent at 120 min. TEM studies reveal that particles are mostly spherical and rod in shape with an average size of $80 \mathrm{~nm}$. Silver nanoparticles prepared with MC as a template have been shown to provide strong SERS enhancement signals of R6G, which can be used as a good Ag-based SERS substrate in the analytical environment for routine measurements.
\end{abstract}

\section{Background}

Surface-enhanced Raman scattering (SERS) has been known and intensely studied for over 50 years. The possibility of utilizing colloidal dispersions of $\mathrm{Ag}$ or $\mathrm{Au}$ in aqueous solutions as a method for enhancing Raman scattering was first demonstrated by Creighton et al. in 1979 [1]. In recent years, with the ever-growing knowledge of plasmonic properties of nanomaterials and the constantly improved engineering capability, SERS has been transformed into a useful analytical technique with some significant advantages for sensitive chemical analysis and interfacial studies [2-5].

It is well known experimentally that the most intense SERS signals are obtained from molecules adsorbed to microscopically rough silver surfaces, such as silver colloids and silver nanoparticles of various shapes [6-9]. Since the last decade a large spectrum of research has been focused to control the size and shape, which is crucial in tuning physical, chemical, and optical properties of nanomaterials. A large variety of synthetic approaches have been explored for the preparation of silver nanoparticles with different morphologies, for example, hydrothermal synthesis [10-12], microemulsion [13, 14], electrochemical deposition [15-17], photochemical method $[18,19], \gamma$-ray irradiation, and so forth [20-22].

Among these approaches "soft" templates are commonly used in order to obtain silver nanoparticles with different morphologies. A range of "soft" templates, such as PVP $[23,24]$, poly (acrylic acid) [25], CTAB [11], SDS [26], DNA [27], have been used to prepare silver nanoparticles. Cellulose is the most important neutral polymer from renewable sources. The aqueous solution of MC contains size-confined, nanosized polls of intermolecular origin $[28,29]$. The polyhydroxylated MC shows dynamic supramolecular association helped by intra- and intermolecular hydrogen bond forming molecular level pools, which act as template for nanoparticle growth. Furthermore, MC is inexpensive, easily obtainable, water-soluble, and easy to wash off. It is a nonionic polymer which contributes to the stabilization of ion concentrations $[30,31]$. 
In this work, we report a simple and effective approach to the aqueous phase synthesis of crystalline silver nanoparticles, which is based on the reduction of silver ions by trisodium citrate in the presence of polymeric stabilizer hydroxylpropyl methyl cellulose (MC). The surface-enhanced Raman scattering of the silver nanoparticles was also investigated.

\section{Materials and Methods}

2.1. Chemicals. Silver nitrate, sodium citrate, R6G, and methyl cellulose (MC) were purchased from Sinopharm Chemical Reagent Beijing Co. Ltd. and were used without further purification. All solutions were prepared with distilled water.

Preparation of silver ammonia chloride: place $150 \mathrm{~mL}$ of water in a $500 \mathrm{~mL}$ beaker. Add $25 \mathrm{~mL} 0.3 \mathrm{M}$ silver nitrate and $25 \mathrm{~mL} 1.0 \mathrm{M}$ sodium chloride to the beaker to form the precipitate. Add $6.0 \mathrm{M}$ ammonia to dissolve the silver chloride and produce the silver ammonia chloride.

2.2. Preparation of Nanoparticles. Definite weight of MC (average molecular weight: 320,000-350,000; degree of polymerization: 1.5-2.0; viscosity: $4000 \mathrm{cps}, 2 \%(\mathrm{w} / \mathrm{v})$ in water at $20^{\circ} \mathrm{C}$ ) was dissolved in $50 \mathrm{~mL}$ of distilled water under stirring. After complete dissolution, temperature of the reaction medium was raised to $75^{\circ} \mathrm{C} .50 \mathrm{~mL}$ silver ammonia chloride solution ( $5 \mathrm{mM}, 10 \mathrm{mM}, 20 \mathrm{mM}$, or $30 \mathrm{mM}$ ) was then added dropwise to MC solution (MC concentrations were $0.1 \%$, $0.15 \%, 0.2 \%, 0.3 \%$, or $0.5 \%$ ) keeping continuous stirring for $30 \mathrm{~min}$. After the mixture was stirred for a given time $(60 \mathrm{~min}$, $90 \mathrm{~min}, 120 \mathrm{~min}$, or $150 \mathrm{~min}$ ), product was washed with water and centrifuged at $5000 \mathrm{rpm}$ for $5 \mathrm{~min}$, and the precipitate was dispersed in $10 \mathrm{~mL}$ of water.

2.3. Testing and Analysis. UV-vis spectra of silver nanoparticles embedded in MC were tested from 200 to $800 \mathrm{~nm}$ by a CARY 50 Spectrophotometer (Hamilton Instrument, Kromatex). A solution containing MC alone was used as a blank.

Size and morphology of synthesized silver nanoparticles were characterized by a JSM-1010 Transmission Electron Microscope (TEM). Samples were prepared by placing a drop of the colloidal solution on a 400 mesh copper grid coated by an amorphous carbon film and evaporating the solvent at room temperature.

To characterize the SERS activity for Ag nanoparticles, the silver colloidal solution was initially concentrated by centrifugation at 5,000 rpm for $5 \mathrm{~min}$, discarding the supernatant and keeping the final concentrated colloids for later use. $5 \mu \mathrm{L}$ of concentrated Ag colloids was then dropped on the surface of a rectangle aluminum plate. Then, $5 \mu \mathrm{L}$ of a $\mathrm{R} 6 \mathrm{G}$ $\left(10^{-7} \mathrm{M}\right)$ or aqueous solution was added into the concentrated Ag colloids to measure SERS spectroscopy. SERS spectra were recorded with a confocal Raman micro-spectrometer (Renishaw, UK) in the range of $300-1800 \mathrm{~cm}^{-1}$ under a $785 \mathrm{~nm}$ diode laser excitation. The laser power is $5 \mathrm{~mW}$. Spectra were collected in backscattering geometry using a microscope equipped with a Leica $20 \times$ objective $(\mathrm{NA}=0.4)$ with a spectral resolution of $2 \mathrm{~cm}^{-1}$; the detection of Raman signal was carried out with a Peltier cooled charge-coupled device (CCD) camera. The software package WIRE 2.0 (Renishaw) was employed for spectral acquisition and analysis. SERS spectra were acquired with a $10 \mathrm{~s}$ integration time. Three spectra were collected to take average from different locations for each sample to ensure representative sampling and incorporate spot-to-spot variability in the signal.

\section{Results and Discussion}

3.1. Effect of MC Concentration. Different concentrations $(0.1 \%, 0.15 \%, 0.2 \%, 0.3 \%$, and $0.5 \%)$ of $\mathrm{MC}$ were used to assist the growth of silver nanoparticles via the reduction of silver ammonia chloride $(10 \mathrm{mM})$ with sodium citrate $(0.1 \%)$ at fixed temperature of $75^{\circ} \mathrm{C}$.

Effect of different concentrations of MC on the shape, size, and dispersity of Ag nanoparticles is truly noticeable from the TEM images (Figure 1). As seen in Figure 1, concentration of $\mathrm{MC}$ (from $0.1 \%$ to $0.5 \%$ ) has significant effect on reduction efficiency, particle shape, and particle size (Figures $1(\mathrm{a})-1(\mathrm{e})$ ). When concentration of MC is $0.1 \%$ (Figure $1(\mathrm{a})$ ), particles are mostly spherical and triangular in shape with an average size of $50 \mathrm{~nm}$. When concentration of $\mathrm{MC}$ is $0.15 \%$ (Figure $1(\mathrm{~b})$ ), particles are mostly spherical and rod in shape with an average size of $60 \mathrm{~nm}$. When the concentration of $\mathrm{MC}$ is $0.2 \%$ (Figure $1(\mathrm{c})$ ), particles are mostly spherical and rod in shape with an average size of $80 \mathrm{~nm}$. When the concentration of MC is $0.3 \%$ (Figure $1(\mathrm{~d})$ ), particles are mostly rod, spherical, triangle, and quadrangle in shape with an average size of $75 \mathrm{~nm}$. When the concentration of MC is $0.5 \%$ (Figure $1(\mathrm{e})$ ), particles are mostly spherical in shape with size ranges from $20 \mathrm{~nm}$ to $100 \mathrm{~nm}$.

UV-vis absorption spectra and SERS spectra of the produced silver nanoparticles were recorded in each case after fixed duration of $120 \mathrm{~min}$ (Figure 2). Figure 2(a) shows UVvis spectra of silver colloid obtained using MC as stabilizing agent. It is clear that the concentration of MC has a great effect on the absorption peak.

Results reveal that the absorption intensity increases initially with the increase in the MC concentration and then reaches a maximum when $\mathrm{MC}$ concentration was $0.3 \%$. A further increase in the $\mathrm{MC}$ concentration results in a decrease in the absorption intensity. This could be ascribed to the high viscosity of MC which represented an impediment to the rate of reducing silver ion by sodium citrate and indicated a tendency towards aggregation. This can be identified by the SEM image in Figure 1(e).

Figure 2(b) shows SERS spectra of silver nanoparticles prepared under different concentrations of MC using Rhodamine $6 \mathrm{G}(\mathrm{R} 6 \mathrm{G})\left(10^{-7} \mathrm{M}\right)$ as a probe molecule. It can be seen that silver nanoparticles have been shown to provide elegant SERS signals of R6G. SERS performance of silver nanoparticles prepared under $0.2 \%$ concentrations of $\mathrm{MC}$ is superior to others. Raman signal can be amplified by adsorbing R6G on the surface of metal nanoparticles and amplification as high as $10^{5}$.

Metallic nanostructures have significant effect on the SERS enhancement. It is well known that Raman enhancement is associated with plasmonic "hot spots", which occur 


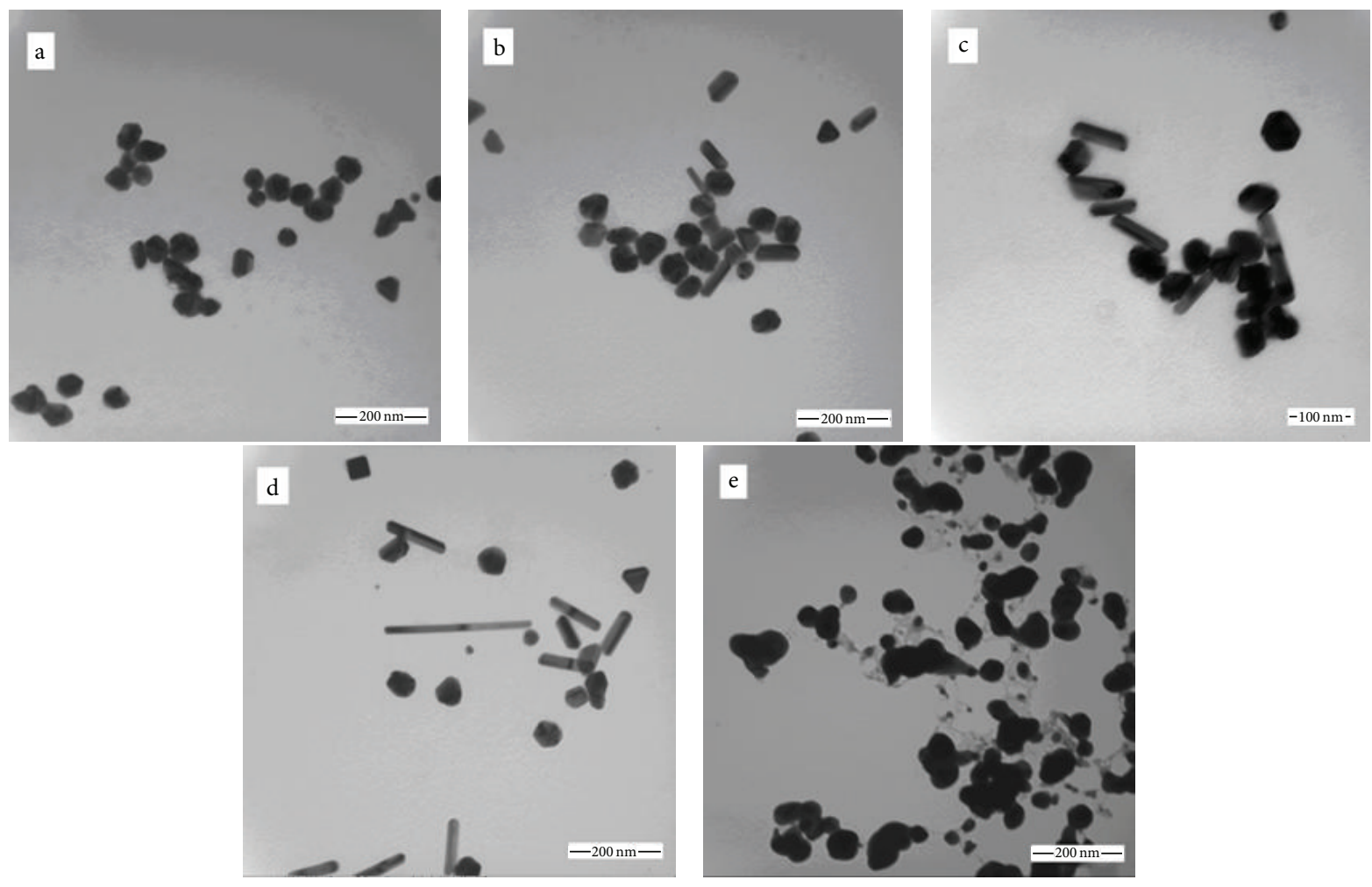

FIGURE 1: SEM images of the products obtained by different concentrations of MC (10 mM silver ammonia chloride, $0.1 \%$ sodium citrate): (a) $0.1 \% \mathrm{MC}$; (b) $0.15 \% \mathrm{MC}$; (c) $0.2 \% \mathrm{MC}$; (d) $0.3 \% \mathrm{MC}$; (e) $0.5 \% \mathrm{MC}$.

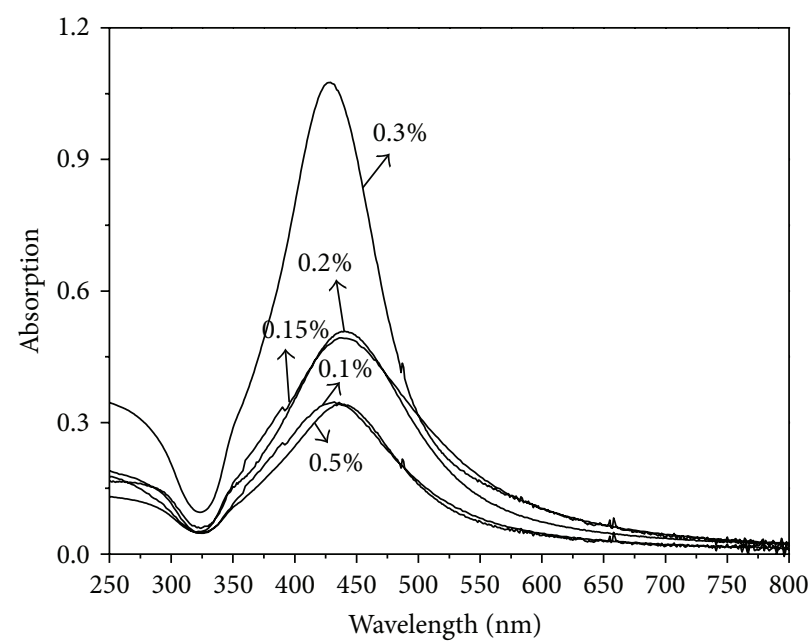

(a)

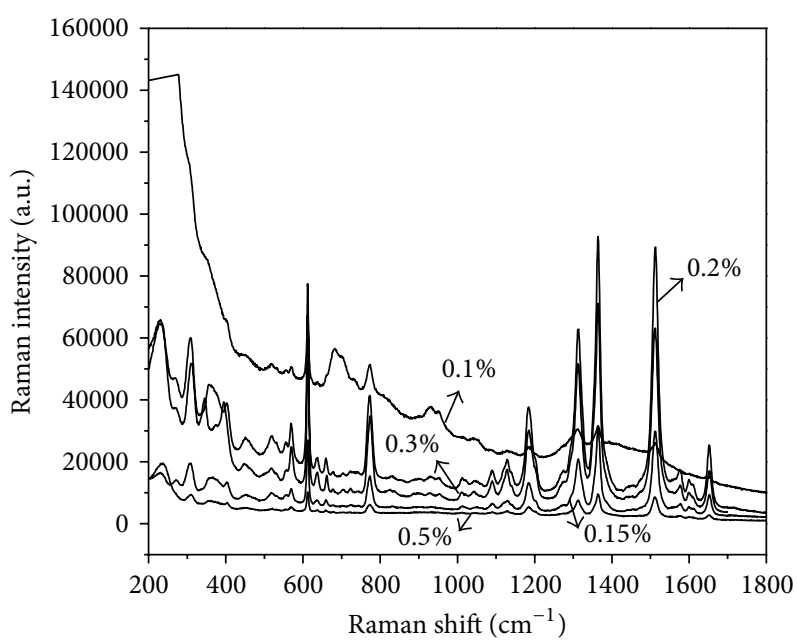

(b)

FIGURE 2: UV-vis spectra and SERS spectra of silver nanoparticles prepared using different MC concentrations. Reaction conditions: $50 \mathrm{~mL}$ of $\mathrm{MC}$ and $0.1 \%$ sodium citrate; $50 \mathrm{~mL} 10 \mathrm{mM}$ silver ammonia chloride; temperature, $75^{\circ} \mathrm{C}$; duration, $120 \mathrm{~min}$.

near the contact point of metallic nanoparticles or nanogaps between two or more particles [32]. The "rough surface" can act as "hot sites" for surface plasma. From the comparison of Raman spectra of R6G $\left(10^{-7} \mathrm{M}\right)$ in Figure 2(b), the Raman enhancement of silver nanoparticles prepared under $0.2 \%$ concentration of MC is better than others. This can be identified by the SEM image in Figure 1(c). As shown in
Figure 1(c), particles are mostly spherical and rod in shape, which easily appear as "rough surface," such as edges, corners, and protuberances [33].

3.2. Concentration of Silver Ammonia Chloride. $0.2 \% \mathrm{MC}$ was used to assist the growth of silver nanoparticles with different concentrations of silver ammonia chloride, namely, $5 \mathrm{mM}$, 

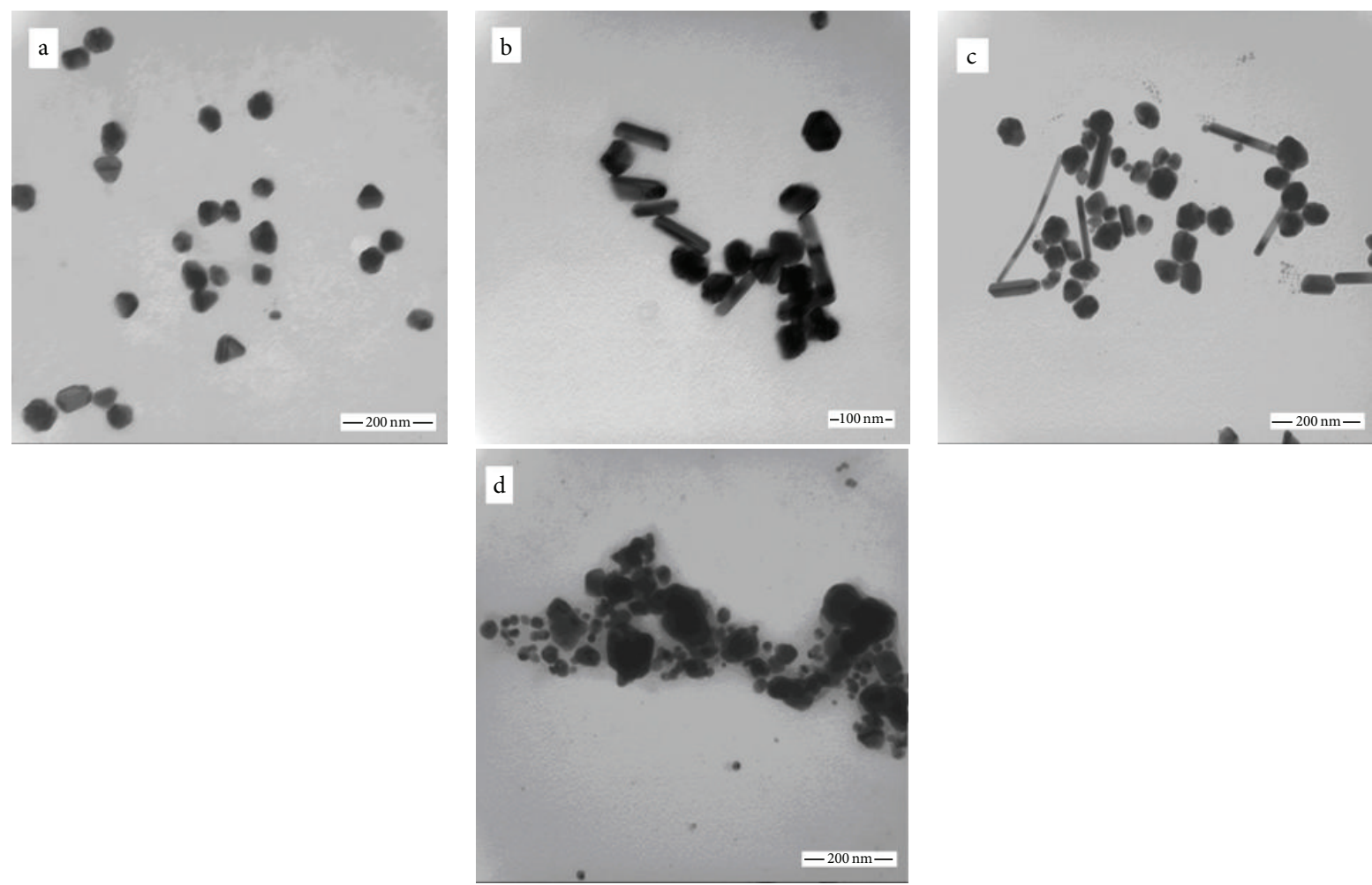

FIGURE 3: SEM images of the products obtained by different concentrations of silver ammonia chloride ( $0.2 \%$ MC, $0.1 \%$ sodium citrate): (a) $5 \mathrm{mM}$; (b) $10 \mathrm{mM}$; (c) $20 \mathrm{mM}$; (d) $30 \mathrm{mM}$.

$10 \mathrm{mM}, 20 \mathrm{mM}$, and $30 \mathrm{mM}$ via the reduction with sodium citrate $(0.1 \%)$ at fixed temperature of $75^{\circ} \mathrm{C}$.

Effect of different concentrations of silver ammonia chloride on the shape, size, and dispersity of Ag nanoparticles is truly noticeable from the TEM images (Figure 3 ).

As seen in Figures 3(a)-3(d), when concentration of silver ammonia chloride is $5 \mathrm{mM}$, particles are mostly spherical in shape with size ranges of $60 \mathrm{~nm}$. With concentration of silver ammonia chloride increasing, particles are mostly rod and spherical in shape with an average size of $75 \mathrm{~nm}$. Further increasing the concentration of silver ammonia chloride to $30 \mathrm{mM}$, particles are mostly spherical in shape with size ranges from $20 \mathrm{~nm}$ to $110 \mathrm{~nm}$.

UV-vis absorption spectra and SERS spectra of the soproduced silver nanoparticles were recorded in each case after fixed duration of $120 \mathrm{~min}$ (Figure 4).

The data in Figure 4(a) shows UV-vis spectra of the silver colloid obtained using MC as stabilizing agent at different silver ammonia chloride concentrations. Results reveal that similar plasmon bands are formed at wavelength $440 \mathrm{~nm}$ with the formation of the ideal bell shape which is characteristic for the formation of silver nanoparticles. Absorption band shifts from $433 \mathrm{~nm}$ to $441 \mathrm{~nm}$ by increasing silver ammonia chloride concentrations from $5 \mathrm{mM}$ to $10 \mathrm{mM}$. This indicated that silver nanoparticles became bigger, which can be identified by the SEM image in Figure 3.

There is a gradual increase in absorption intensity, by increasing silver ammonia chloride concentration up to 10 $\mathrm{mM}$. Further increasing silver ammonia chloride concentration, absorption intensity decreased, the plasmon band is broadened, and simple test for silver ion using $\mathrm{NaCl}$ solution indicates low conversion of silver ions to metallic silver nanoparticles. When silver ammonia chloride concentrations increased to $30 \mathrm{mM}$, the absorption intensity decreased and exhibited a broad band, which indicated the formation of silver nanoparticles with higher aggregation. These results are in agreement with the SEM image in Figure 3(d).

The data in Figure 4(b) shows SERS spectra of silver nanoparticles synthesized with different silver ammonia chloride concentrations using $\mathrm{R} 6 \mathrm{G}\left(10^{-7} \mathrm{M}\right)$ as a probe molecule. Results reveal that silver ammonia chloride concentrations have significant effect on the enhancement performance of silver nanoparticles. Silver nanoparticles prepared under $20 \mathrm{mM}$ silver ammonia chloride have been shown to provide SERS signals of R6G which are stronger than others.

Although the surface of silver nanoparticles prepared under $20 \mathrm{mM}$ silver ammonia chloride showed "rougher" and the Raman signal is stronger than others, the baseline is not as good as silver nanoparticles prepared under $10 \mathrm{mM}$. Considering the size and morphology of nanoparticles, the optimum silver ammonia chloride concentration was $10 \mathrm{mM}$.

3.3. Effect of the Reducing Agent. Effect of different concentrations of sodium citrate on the shape, size, and dispersity of $\mathrm{Ag}$ nanoparticles is truly noticeable from the TEM images (Figure 5). Figure 5 reveals that the number of Ag nanoparticles increases by increasing the concentration of sodium citrate from $0.05 \%$ to $0.3 \%$. 


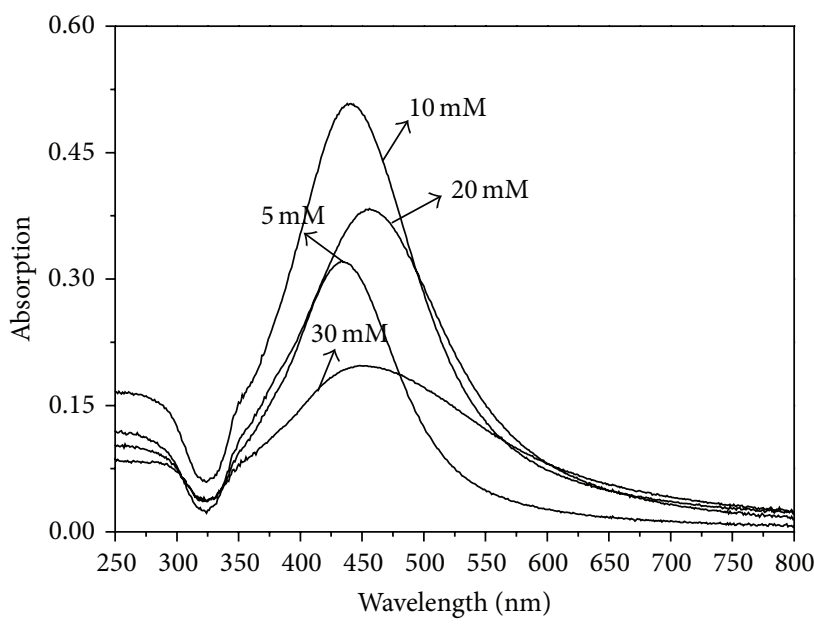

(a)

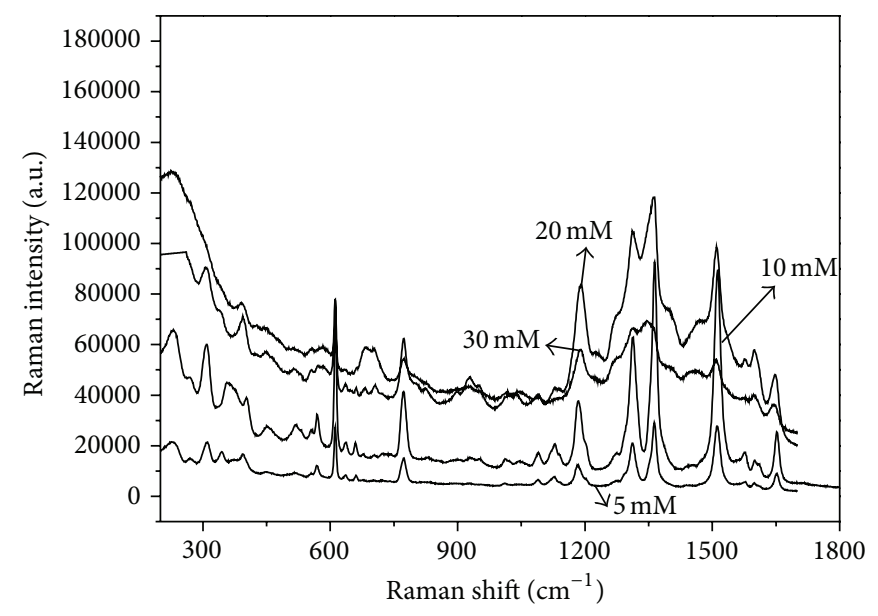

(b)

FIGURE 4: UV-vis spectra and SERS spectra of silver nanoparticles prepared using different silver ammonia chloride concentrations. Reaction conditions: $50 \mathrm{~mL}$ of $0.2 \% \mathrm{MC}$ and $0.1 \%$ sodium citrate; $50 \mathrm{~mL}$ silver ammonia chloride; temperature, $75^{\circ} \mathrm{C}$; duration, $120 \mathrm{~min}$.
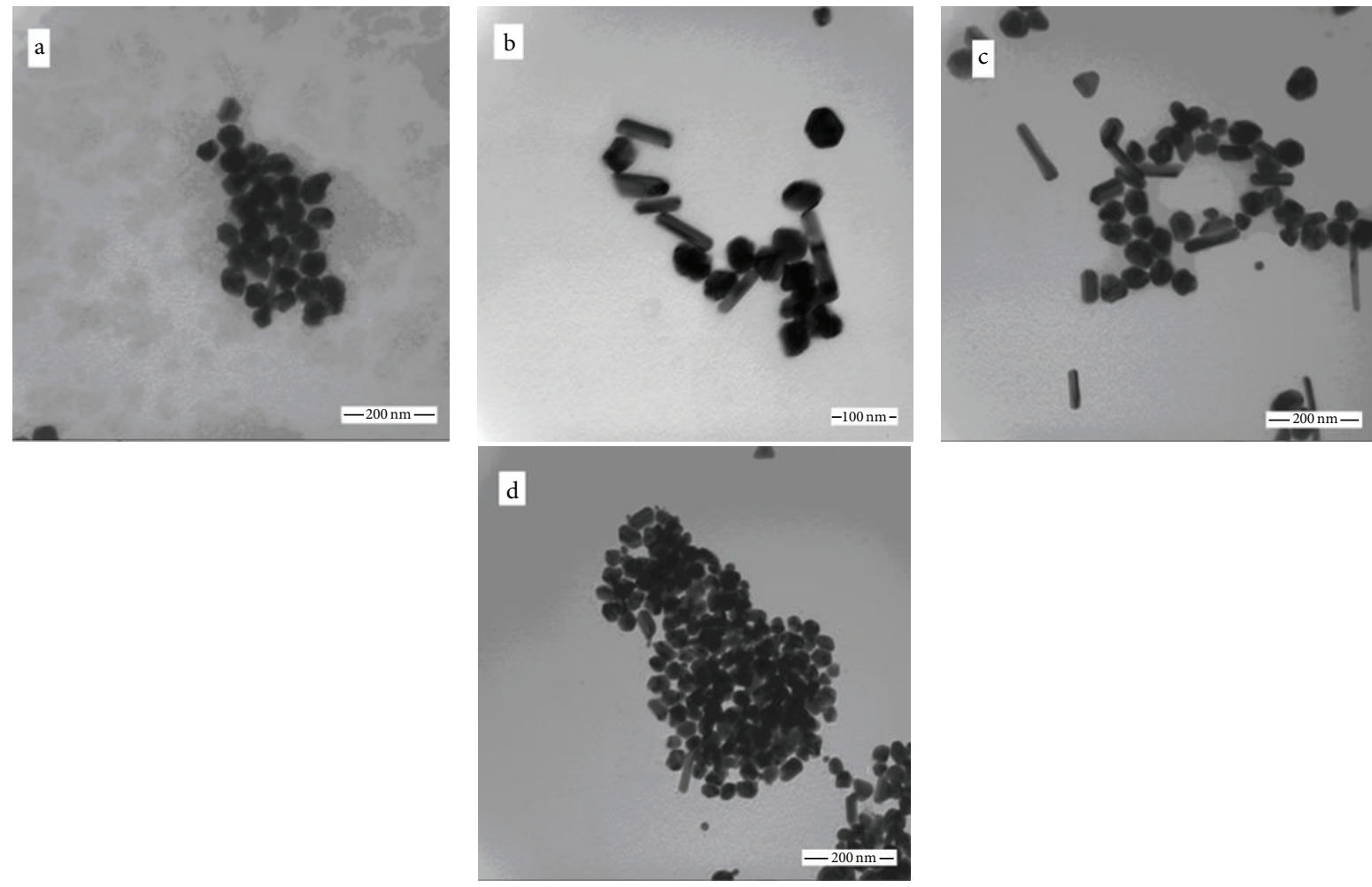

FIGURE 5: SEM images of the products obtained by different concentrations of sodium citrate (10 mM silver ammonia chloride, $0.2 \% \mathrm{MC})$ : (a) $0.05 \%$ sodium citrate; (b) $0.1 \%$ sodium citrate; (c) $0.2 \%$ sodium citrate; (d) $0.3 \%$ sodium citrate.

Figure 6 shows UV-vis absorption spectra and SERS spectra of silver nanoparticle prepared at different concentrations of sodium citrate $(0.05 \%, 0.1 \%, 0.2 \%$, or $0.3 \%)$, with $0.2 \% \mathrm{MC}$ and $75^{\circ} \mathrm{C}$ after fixed duration of $120 \mathrm{~min}$

The experiments indicated that the concentration of sodium citrate played a significant role in the formation of the silver nanoparticles. It is clear that there is a gradual increase in the absorption intensity by increasing the sodium citrate concentration, which could be ascribed to amounts of silver ion reduced and used for cluster formation. With increasing the sodium citrate concentration, the absorption band shifts from $440 \mathrm{~nm}$ to $455 \mathrm{~nm}$, which indicated that the diameter of silver nanoparticles became bigger.

To demonstrate the enhancement of Raman spectral intensity for the silver nanoparticles substrate, SERS spectra of R6G on silver nanoparticles are shown in Figure 6(b). 


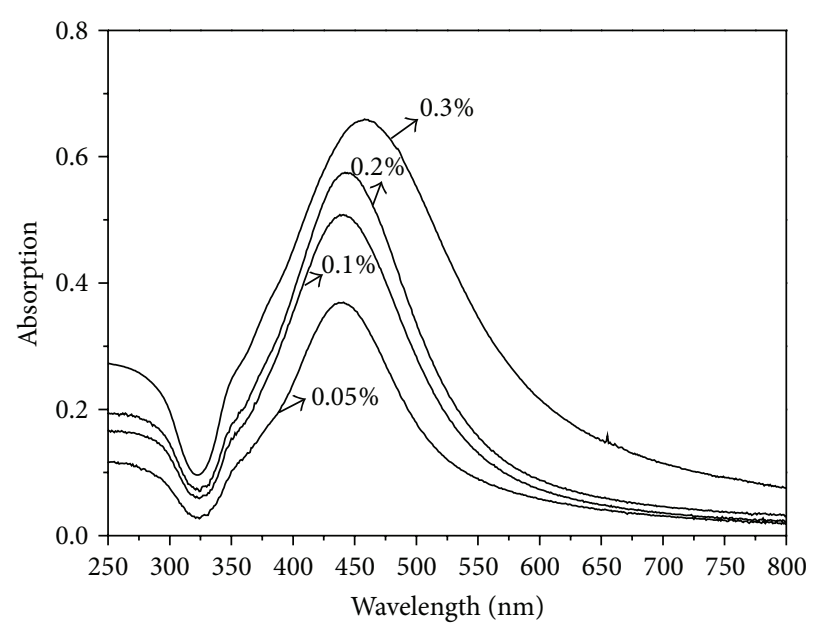

(a)

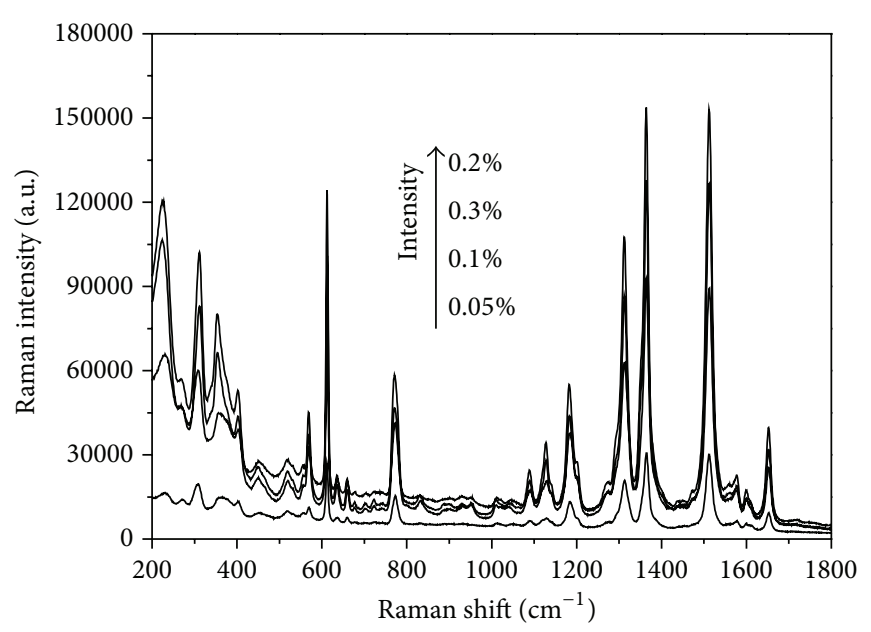

(b)

FIGURE 6: UV-vis spectra and SERS spectra of silver nanoparticles prepared using different sodium citrate concentrations. Reaction conditions: $50 \mathrm{~mL}$ of $0.2 \% \mathrm{MC} ; 50 \mathrm{~mL} 10 \mathrm{mM}$ silver ammonia chloride; temperature, $75^{\circ} \mathrm{C}$; duration, $120 \mathrm{~min}$.

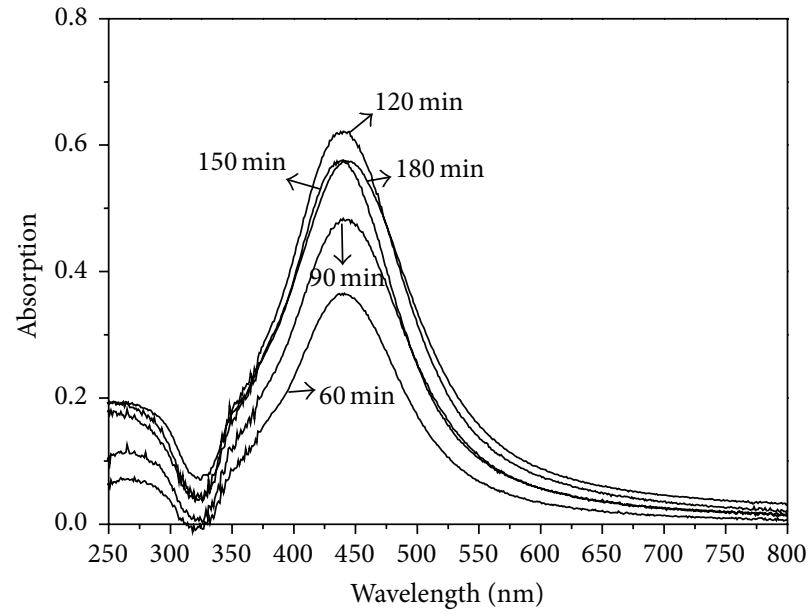

(a)

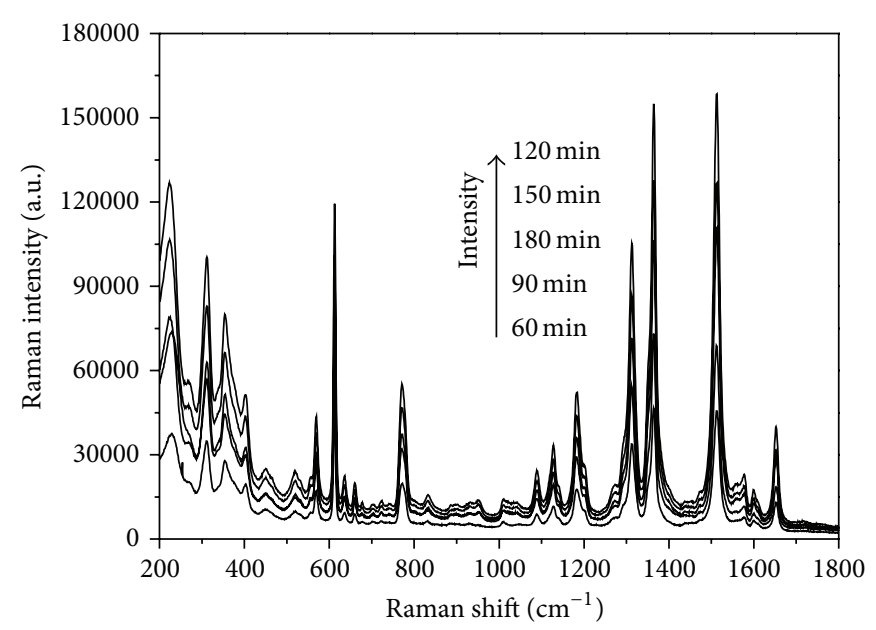

(b)

FIGURE 7: UV-vis spectra and SERS spectra of silver nanoparticles prepared at different durations. Reaction conditions: $50 \mathrm{~mL}$ of $0.2 \% \mathrm{MC}$ and $0.2 \%$ sodium citrate; $50 \mathrm{~mL} 10 \mathrm{mM}$ silver ammonia chloride; temperature, $75^{\circ} \mathrm{C}$.

We found remarkable enhancements in the SERS spectrum of $\mathrm{R} 6 \mathrm{G}$ adsorbed on the silver nanoparticles prepared at $0.2 \%$ concentrations of sodium citrate compared with those adsorbed on the other silver nanoparticles.

The "rough surface" of silver nanoparticles can act as "hot sites" for surface plasma. The silver nanoparticles show stronger Raman enhancement with rougher surface of silver nanoparticles. Results show that the density of silver nanoparticles also has a significant effect on the Raman enhancement. The optimum concentration of sodium citrate is $0.2 \%$.

3.4. Effect of Reaction Duration. Preparation of silver nanoparticles was carried out at $75^{\circ} \mathrm{C}$ with $0.2 \% \mathrm{MC}$, and samples from the reaction medium were withdrawn at different time intervals, namely, $60 \mathrm{~min}, 90 \mathrm{~min}, 120 \mathrm{~min}, 150 \mathrm{~min}$, and $180 \mathrm{~min}$, for recording the UV-vis absorption spectra and the SERS spectra of the formed silver nanoparticles at these time intervals (Figure 7).

The data in Figure 7(a) reveal that there is a gradual increase in the absorption intensity by increasing reaction duration up to $120 \mathrm{~min}$. When the reaction duration is $60 \mathrm{~min}$, the plasmon band is broadened, which indicates that conversion rate of silver ions to metallic silver nanoparticles is low. When the reaction duration is more than $90 \mathrm{~min}$, the plasmon intensity leads to outstanding enhancement, which indicates that large amounts of silver ions are reduced and used. Further prolonging reaction duration, absorption intensity decreased. Absorption band shifts from $440 \mathrm{~nm}$ to $446 \mathrm{~nm}$, which indicates higher aggregation of silver nanoparticles.

The data in Figure 7(b) reveal that the silver nanoparticles have been shown to provide elegant SERS signals of R6G. The Raman signal can be amplified by adsorbing the R6G on 
the surface of metal nanoparticles and amplification as high as $10^{5}$. SERS performance of silver nanoparticles prepared at $120 \mathrm{~min}$ is superior to others. Based on the above, optimum duration for the preparation of silver nanoparticles is $120 \mathrm{~min}$. These results are in agreement with the expected data obtained from the UV-vis spectra (Figure 7(a)).

\section{Conclusions}

In summary, a good Ag-based SERS substrate has been prepared by a simple and low-cost method with MC as a template. Results show that the MC concentration and reducing agent play a crucial role in performance of silver nanoparticles. Optimum preparation conditions of synthesis of SERS substrates were as follows: $10 \mathrm{mM}$ silver ammonia chloride and $0.2 \%$ concentration of $\mathrm{MC}$ at $75^{\circ} \mathrm{C}$, then reducing in $0.2 \%$ reducing agent at $120 \mathrm{~min}$. Silver nanoparticles prepared with $\mathrm{MC}$ as a template have more remarkable enhancements in the SERS spectrum of R6G, which can be used as a good Ag-based SERS substrate in the analytical environment for routine measurements.

\section{Acknowledgments}

This work was financially supported by the Natural Science Foundation of Fujian Province in China (no. 2011J01288) and the Maior Science and Technology Projects of Fujian Province in China (2008HZ00011).

\section{References}

[1] J. A. Creighton, C. G. Blatchford, and M. G. Albrecht, "Plasma resonance enhancement of Raman scattering by pyridine adsorbed on silver or gold sol particles of size comparable to the excitation wavelength," Journal of the Chemical Society, Faraday Transactions, vol. 75, pp. 790-798, 1979.

[2] D. A. Schultz, "Plasmon resonant particles for biological detection," Current Opinion in Biotechnology, vol. 14, no. 1, pp. 13-22, 2003.

[3] L. Brus, "Noble metal nanocrystals: plasmon electron transfer photochemistry and single-molecule raman spectroscopy," Accounts of Chemical Research, vol. 41, no. 12, pp. 1742-1749, 2008.

[4] J. N. Anker, W. P. Hall, O. Lyandres, N. C. Shah, J. Zhao, and R. P. van Duyne, "Biosensing with plasmonic nanosensors," Nature Materials, vol. 7, no. 6, pp. 442-453, 2008.

[5] H. Jans and Q. Huo, "Gold nanoparticle-enabled biological and chemical detection and analysis," Chemical Society Reviews, vol. 41, no. 7, pp. 2849-2866, 2012.

[6] K. Kim, K. L. Kim, D. Shin, J.-Y. Choi, and K. S. Shin, "Surfaceenhanced Raman scattering of 4-aminobenzenethiol on Ag and Au: PH dependence of b2-type bands," The Journal of Physical Chemistry C, vol. 116, no. 7, pp. 4774-4779, 2012.

[7] C. Viets and W. Hill, "Single-fibre surface-enhanced Raman sensors with angled tips," Journal of Raman Spectroscopy, vol. 31, pp. 625-631, 2000.

[8] M. A. Mahmoud and M. A. El-Sayed, "Different plasmon sensing behavior of silver and gold nanorods," The Journal of Physical Chemistry Letters, vol. 4, no. 9, pp. 1541-1545, 2013.
[9] C. J. Murphy, T. K. Sau, C. J. Orendorff, and A. M. Gole, "Surface enhanced Raman spectroscopy using shaped gold nanoparticles," Nanotechnology, vol. 23, no. 11, Article ID 115501, 2012.

[10] J. A. Dieringer, R. B. Lettan II, K. A. Scheidt, and R. P. Van Duyne, "A frequency domain existence proof of singlemolecule surface-enhanced Raman spectroscopy," Journal of the American Chemical Society, vol. 129, no. 51, pp. 16249-16256, 2007.

[11] P. D. Jadzinsky, G. Calero, C. J. Ackerson, D. A. Bushnell, and R. D. Kornberg, "Structure of a thiol monolayer-protected gold nanoparticle at 1.1 Å resolution," Science, vol. 318, no. 5849, pp. 430-433, 2007.

[12] J. Yang and J. Pan, "Hydrothermal synthesis of silver nanoparticles by sodium alginate and their applications in surfaceenhanced Raman scattering and catalysis," Acta Materialia, vol. 60, pp. 4753-4758, 2012.

[13] Y. Wang, K. Lee, and J. Irudayaraj, "Silver nanosphere SERS probes for sensitive identification of pathogens," The Journal of Physical Chemistry C, vol. 114, no. 39, pp. 16122-16128, 2010.

[14] R. Saha, S. Rakshit, D. Majumdar, A. Singha, R. K. Mitra, and S. K. Pal, "Nanostructure, solvation dynamics, and nanotemplating of plasmonically active SERS substrate in reverse vesicles," Journal of Nanoparticle Research, vol. 15, pp. 1-12, 2013.

[15] R. Oriňáková, L. Škantárová, A. Oriňák, J. Demko, M. Kupková, and J. T. Andersson, "Electrochemical deposition of SERS active nanostructured silver films," International Journal of Electrochemical Science, vol. 8, pp. 80-99, 2013.

[16] V. Halouzka, P. Jakubec, L. Kvitek et al., "Deposition of nanostructured Ag films on silicon wafers by electrochemical/electrophoretic deposition for electrochemical and SERS sensing," Journal of the Electrochemical Society, vol. 160, pp. B54-B59, 2013.

[17] Y. Zhang, S. Sun, D. Deng, X. Song, B. Ding, and Z. Yang, "Electrochemical deposition mediated growth of hierarchical $\mathrm{Au}$ architectures and the applications for SERS," CrystEngComm, vol. 14, no. 2, pp. 656-662, 2012.

[18] K.-H. Yang and C.-M. Chang, "Using a photochemical method and chitosan to prepare surface-enhanced Raman scatteringactive silver nanoparticles," Analytica Chimica Acta, 2012.

[19] A. Pal and T. Pal, "Silver nanoparticle aggregate formation by a photochemical method and its application to SERS analysis," Journal of Raman Spectroscopy, vol. 30, no. 3, pp. 199-204, 1999.

[20] S.-H. Choi and H. G. Park, "Surface-enhanced Raman scattering (SERS) spectra of sodium benzoate and 4-picoline in Ag colloids prepared by $\gamma$-irradiation," Applied Surface Science, vol. 243, no. 1-4, pp. 76-81, 2005.

[21] H. T. Tung, I. G. Chen, I. M. Kempson et al., "Shape-controlled synthesis of silver nanocrystals by X-ray irradiation for inkjet printing," ACS Applied Materials \& Interfaces, vol. 4, pp. 59305935, 2012.

[22] M. Moskovits, "Surface-enhanced Raman spectroscopy: a brief retrospective," Journal of Raman Spectroscopy, vol. 36, no. 6-7, pp. 485-496, 2005.

[23] N. Vijayakumar, E. Subramanian, and D. P. Padiyan, "Conducting polyaniline blends with the soft template poly (vinyl pyrrolidone) and their chemosensor application," International Journal of Polymeric Materials, vol. 61, pp. 847-863, 2012.

[24] X.-L. Tang, P. Jiang, G.-L. Ge, M. Tsuji, S.-S. Xie, and Y.J. Guo, "Poly(N-vinyl-2-pyrrolidone) (PVP)-capped dendritic gold nanoparticles by a one-step hydrothermal route and their high SERS effect," Langmuir, vol. 24, no. 5, pp. 1763-1768, 2008. 
[25] L. Jiang, Z. Wu, D. Wu, W. Yang, and R. Jin, "Controllable embedding of silver nanoparticles on silica nanospheres using poly(acrylic acid) as a soft template," Nanotechnology, vol. 18, no. 18, Article ID 185603, 2007.

[26] J. H. Kim, B. R. Min, J. Won, and Y. S. Kang, "Effect of the polymer matrix on the formation of silver nanoparticles in polymer-silver salt complex membranes," Journal of Polymer Science B, vol. 44, no. 8, pp. 1168-1178, 2006.

[27] L.-B. Yang, G.-Y. Chen, J. Wang, T.-T. Wang, M.-Q. Li, and J.H. Liu, "Sunlight-induced formation of silver-gold bimetallic nanostructures on DNA template for highly active surface enhanced Raman scattering substrates and application in TNT/tumor marker detection," Journal of Materials Chemistry, vol. 19, no. 37, pp. 6849-6856, 2009.

[28] P. Sarkar, D. K. Bhui, H. Bar et al., "Aqueous-phase synthesis of silver nanodiscs and nanorods in methyl cellulose matrix: photophysical study and simulation of UV-Vis extinction spectra using DDA method," Nanoscale Research Letters, vol. 5, no. 10, pp. 1611-1618, 2010.

[29] P. Raveendran, J. Fu, and S. L. Wallen, "Completely "Green" synthesis and stabilization of metal nanoparticles," Journal of the American Chemical Society, vol. 125, no. 46, pp.13940-13941, 2003.

[30] T. Thongtem, C. Pilapong, and S. Thongtem, "Solvothermal synthesis of CdS nanorods using hydroxyethyl cellulose as a template," Current Applied Physics, vol. 9, no. 6, pp. 1272-1277, 2009.

[31] I. Arvanitoyannis and C. G. Biliaderis, "Physical properties of polyol-plasticized edible blends made of methyl cellulose and soluble starch," Carbohydrate Polymers, vol. 38, no. 1, pp. 47-58, 1999.

[32] J. Zhang, X. Li, X. Sun, and Y. Li, "Surface enhanced Raman scattering effects of silver colloids with different shapes," The Journal of Physical Chemistry B, vol. 109, no. 25, pp. 1254412548, 2005.

[33] M. Potara, D. Maniu, and S. Astilean, "The synthesis of biocompatible and SERS-active gold nanoparticles using chitosan," Nanotechnology, vol. 20, no. 31, Article ID 315602, 2009. 

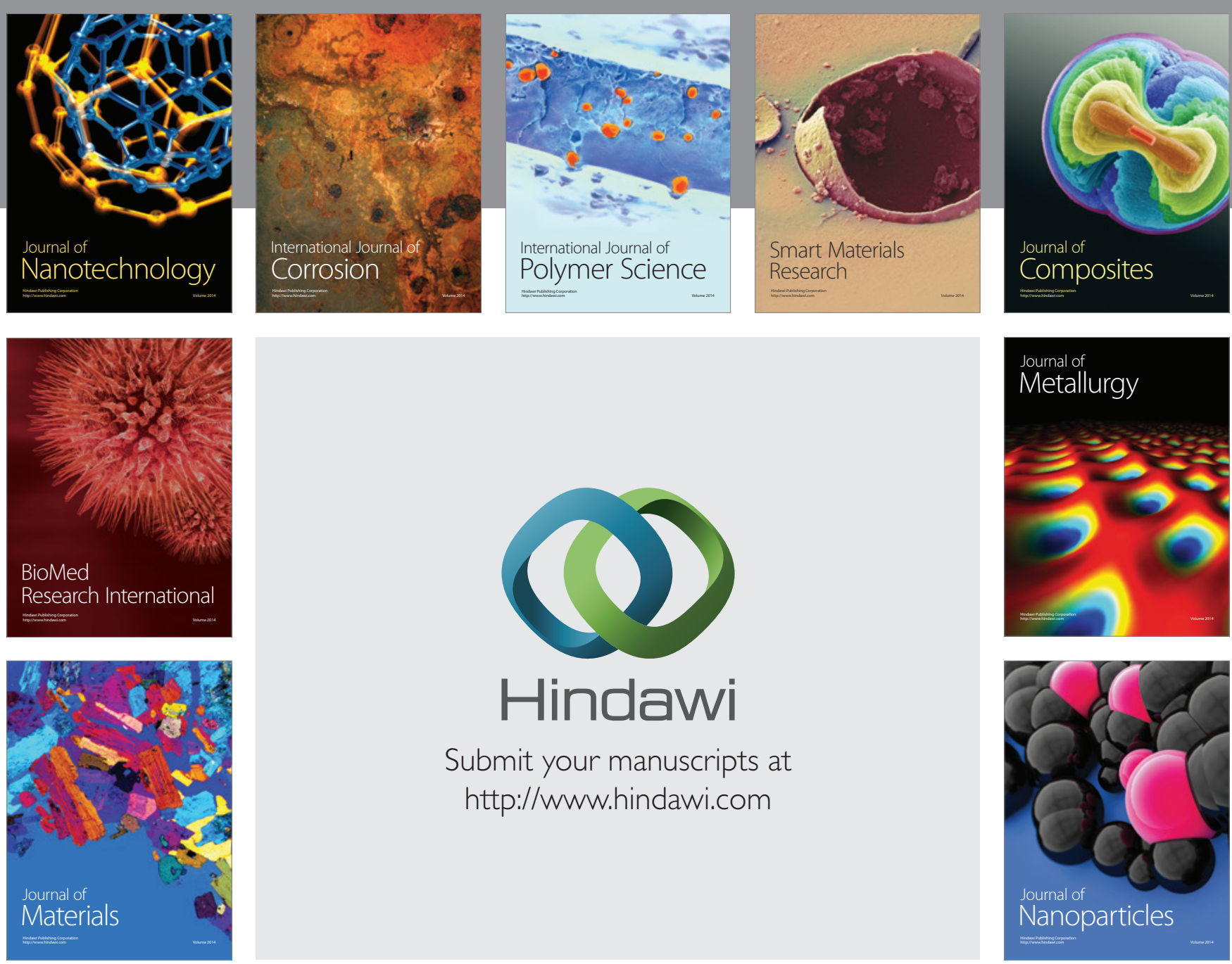

Submit your manuscripts at http://www.hindawi.com
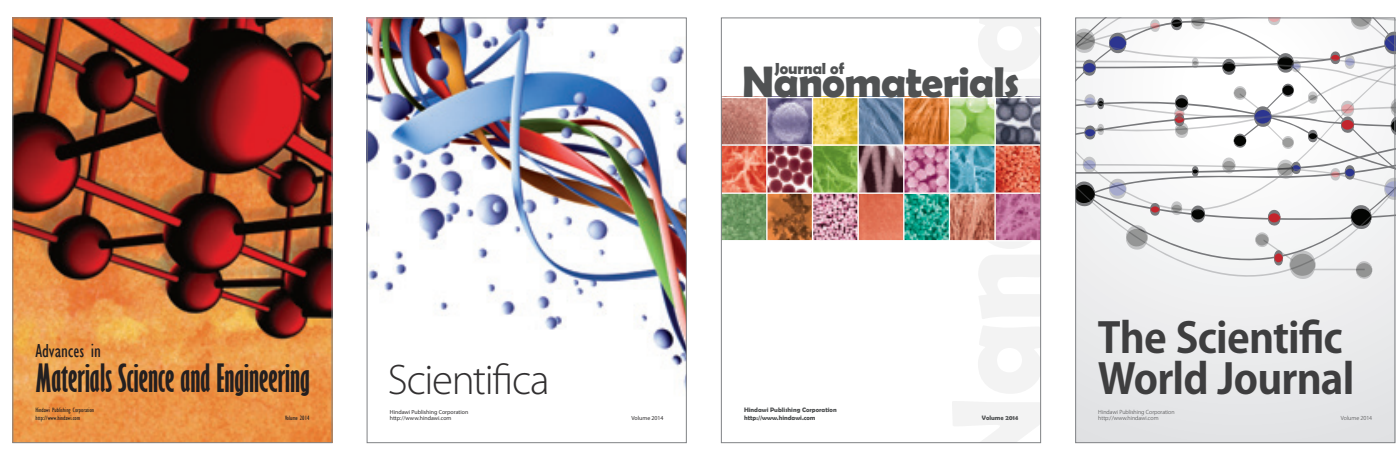

\section{The Scientific World Journal}
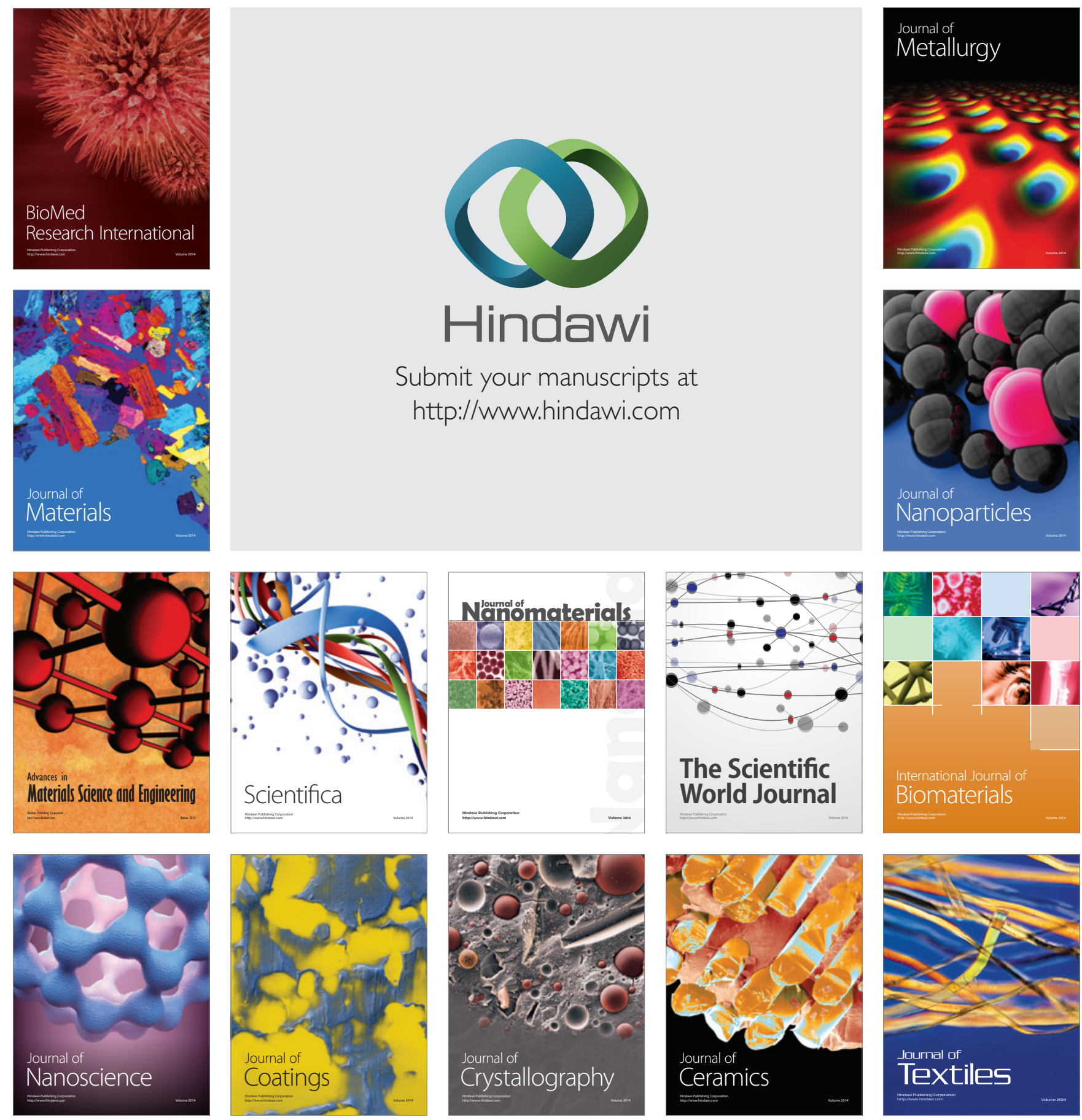